\title{
The Gravitational Force Quantum and its Value
}

\author{
Tomas Kala \\ Faculty of Informatics and Management, University of Hradec Kralove, 500 03, Czech Republic
}

\begin{abstract}
Gravitation is one of the basic phenomena of the world. Tremendous number of theoretical works on origin, nature, essentials, consequences, etc. of the gravitation and related phenomena were published so far. The most prominent ones are based on the Albert Einstein's general theory of relativity. The author of this communication based his approach to the gravitation on Isaac Newton's law of the universal gravitation and related quantities, i.e. gravitational forces of matter objects, distance and motion. Namely on the fact, that the gravitation force is - as well as the inertia, mass, space "occupied" and other properties are - principal features/attributes/properties of matter objects. Gravitation is an additive property of matter objects. Taking into account other positivistic quantities like mass of the Earth, standard acceleration of gravity, and the value of the atomic unit of mass, the author defined a gravitational force of atomic unit (or "the Gravitational Force Quantum”) as a gravitational force which exerts one atomic unit of Earth's mass on 1 kilogram of a mass on Earth's surface, and he calculated its value: GFO $=1.4958 \times 10^{-54} \mathrm{~N}$. This quantity can be useful for further development of the "quantum mechanical" approach to the description and general notion about the world.
\end{abstract}

Key words: Gravitation, gravitational force of atomic unit, gravitational force quantum.

\section{Nomenclature}

$\begin{array}{ll}m: & \text { Mass. } \\ F: & \text { Gravitational force }-9.80665 \mathrm{~N}[1] . \\ K g f: & \text { Kilogram-force }-9.80665 \mathrm{~N} \mathrm{[1].} \\ M: & \text { Mass of the Earth }-5.97219 \times 10^{24} \mathrm{~kg} \mathrm{[1].} \\ \text { AUM: } & \text { Atomic unit of mass }-9.1093829 \times 10^{-31} \mathrm{~kg} \mathrm{[2]} . \\ N_{\text {AUM }}: & \text { Number of atomic units of mass of Earth. } \\ \text { GFO: } & \text { A gravitational force quantum. }\end{array}$

\section{Introduction}

Gravitation is one of the basic phenomena of the world [3, 4]. In Wikipedia [5], gravity or gravitation is defined as a natural phenomenon by which all things attract one another including stars, planets, galaxies and even light and sub-atomic particles.

Gravity is a cause of time dilation (time lapses more slowly in strong gravitation).

On Earth, gravity gives weight to physical objects and causes the tides. Gravity has an infinite range, and it cannot be absorbed, transformed, or shielded against.

Gravity is described by the general theory of relativity, proposed by Albert Einstein in 1915 [6-8].

Corresponding author: Tomas Kala, DrSc, assistent professor, research fields: material sciences and social sciences. E-mail: tomas.kala@uhk.cz.
The theory describes gravity, not as a force, but as a consequence of the curvature of the space-time caused by the uneven distribution of mass/energy. For most applications, gravity is well approximated by Newton's law of universal gravitation, which postulates that the gravitational force of two bodies of mass is directly proportional to the product of their masses and inversely proportional to the square of the distance between them.

Gravity is the weakest of the four fundamental interactions of nature. The gravitational attraction is approximately $10^{-38}$ times the strength of the strong force (i.e. gravity is 38 orders of magnitude weaker), $10^{-36}$ times the strength of the electromagnetic force, and $10^{-29}$ times the strength of the weak force.

Very interesting are also theories on the Quantum Gravity and related approaches to the phenomenon of Gravitation [9].

The author of this communication based his approach to the gravitation on Isaac Newton's law of the universal gravitation and related quantities, i.e. gravitational forces of mass, distance and motion [3-5, 10]. Namely on the fact, that the gravitation force is as well as the inertia, mass, space "occupied" and 
others are - a principal/immanent feature/attribute/property of a matter objects ${ }^{1}$. Gravitation is an additive property/attribute/trait of any of matter objects. The more mass a matter object has, the more gravitation it exhibits.

Taking into account other positivistic quantities like mass of the Earth, standard acceleration of gravity, and a value of the atomic unit of mass, the author defined a gravitational force of atomic unit (or "the Gravitational Force Quantum”) and calculated its value.

\section{Methodology}

According to the Newton's law, a mass of $\mathrm{m}=1 \mathrm{~kg}$ exerts on Earth's surface a gravitational force $\mathrm{F}=$ $9.80665 \mathrm{~N}$ (or 1.0 kilogram-force, $1 \mathrm{kgf}=9.80665 \mathrm{~N}$ by definition).

The mass of the Earth $\left(5.97219 \times 10^{24} \mathrm{~kg}\right.$ [1] $)$ can be expressed as:

$$
\mathrm{M}=\mathrm{AUM} \times \mathrm{N}_{\mathrm{AUM}}
$$

where, AUM is an atomic unit of mass, $9.10938291 \times$ $10^{-31} \mathrm{~kg}$ [2]), $\mathrm{N}_{\mathrm{AUM}}$ is a number of atomic units of mass contained in the Earth, i.e.:

$$
\begin{aligned}
\mathrm{N}_{\text {AUM }}=\mathrm{M} / \mathrm{AUM} & =5.97219 \times 10^{24} / 9.10938291 \times \\
10^{-31} & =0.6556 \times 10^{55}
\end{aligned}
$$

The value of gravitational force attributed to one atomic unit of (Earth's) mass, i.e. a “GFQ (Gravitational force quantum)” can be calculated as:

$$
\begin{aligned}
\mathrm{GFQ}=\mathrm{F} / \mathrm{NAUM} & =9.80665 / 0.6556 \times 10^{55} \mathrm{~N} \\
& =1.4958 \times 10^{-54} \mathrm{~N}
\end{aligned}
$$

\section{Results and Discussion}

The GFQ, defined by the author as a gravitational force which exerts one atomic unit of Earth's mass on 1

\footnotetext{
${ }^{1}$ Difference between matter and mass in author's approach is as follows: Matter is anything that has mass and takes up space and other properties, like inertia, gravitation, ability to change/modify fields, space-time structures and dimensions, particle structure, electric charges, electric, magnetic and electromagnetic properties and phenomena, attractive and repulsive forces, mechanical properties of matter, storing and conversions of energy, and phenomena which are intrinsic to all the physical, inorganic, organic, living, thinking etc. objects and/or phenomena in Nature and World [12].
}

kilogram of a mass on Earth's surface, has a value $1.4958 \times 10^{-54} \mathrm{~N}$.

Verity of the value of the GFO might be limited by correctness of the following values: the atomic unit of mass $9.10938291 \times 10^{-31} \mathrm{~kg}$, the $\mathrm{kgf}=9.80665 \mathrm{~N}$ and the Earth's mass $5.97219 \times 10^{24} \mathrm{~kg}$, which were used in the calculation of the gravitational force quantum. It can be foreseen that the value of GFO will be revised in future development of notion.

\section{Conclusions}

Gravitation/gravity should be considered as a immanent property of the matter, like:

- Mass or weight.

- Inertia.

- Space/volume.

- Structure.

- Components/parts/particles.

- Transformability.

- Electrical, dielectric, ferroelectric, piezoelectric, pyroelectric, superconductive, etc. properties/parameters.

- Magnetic, diamagnetic, ferromagnetic, antiferromagnetic, and others - magnetic properties/parameters.

- Electromagnetic properties/parameters.

- Optical, electro-optical, magneto-optical, acousto-optical, and others - optical properties/parameters.

- Mechanical, electro-mechanical, acousto-mechanical, piezo-mechanical, and others -mechanical properties/parameters.

- Thermal, thermomechanical, thermoplastic and related properties/parameters.

- Chemical, crystallo-chemical, biochemical, and others related properties/parameters.

- Biological, microbiological, macro-biological, environmental and others life related properties/parameters.

- Many other properties and parameters. 
Respecting the obvious fact, that gravitation/gravity is an additive property of matter objects and taking into account other positivistic quantities like mass of the Earth, standard acceleration of gravity, and a value of the atomic unit of mass, the author defined a gravitational force of atomic unit (or "the Gravitational Force Quantum”) as a gravitational force which exerts one atomic unit of Earth's mass on $1 \mathrm{~kg}$ of a mass on Earth's surface, and he calculated its value: $\mathrm{GFO}=1.4958 \times 10^{-54} \mathrm{~N}$.

This quantity can be useful for further development of the "quantum mechanical" approach to the description and general notion about the world.

It is an opinion of the author, that physics might be clarified if gravitation would be excluded from a group of the fundamental forces of Nature, i.e. weak, electromagnetical, and strong forces. The gravitation force should be considered on the same "rank" of forces like attractive/repulsive electrical, magnetic or other forces.

Gravitation might perhaps be connected/caused/ explained by distortion of the space around/because of material objects. For further details see [12].

\section{References}

[1] NASA. "Earth: by the Numbers.” Accessed Sep. 3, 2015.
http://solarsystem.nasa.gov/planets/profile.cfm?Object=E arth\&Display=Facts.

[2] NIST. "Fundamental Physical Constants - Complete Listing.” $\quad$ Accessed Sep. 3, 2015. http://physics.nist.gov/cuu/Constants/Table/allascii.txt.

[3] Halliday, D. and Resnick, R. 1981. "Fundamentals of Physics.” $2^{\text {nd }}$ Edition, John Wiley\&"Sons, Inc. N.Y., Chichester, Brisbane, Toronto. ISBN 0-471-03363-4.

[4] Cook, A. H. “Gravity Physics.” Encyklopaedia Britannica, $\begin{array}{llll}\text { Accessed } & \text { Sep. } & 3015 .\end{array}$ http://www.britannica.com/science/gravity-physics.

[5] Wikipedia. "Gravity.” Accessed Sep. 3, 2015. https://en.wikipedia.org/wiki/Gravity.

[6] Einstein, A. 1915. "Die Feldgleichungen der Gravitation.” In Sitzungsberichte der Preussischen Akademie der Wissenschaften zu Berlin.

[7] Einstein, A. 1916. "Die Grundlage der Allgemeinen Relativitätstheorie.” Annalen der Physik 49: 769-822.

[8] Einstein, A. 1917. "Kosmologische Betrachtungen zur Allgemeinen Relativitätstheorie.” In Sitzungsberichte der Preußischen Akademie der Wissenschaften.

[9] Weinstein, S. "Quantum Gravity.” Stanford Encyklopedia of Philosophy. Accessed Sep. 3, 2015. http://plato.stanford.edu/entries/quantum-gravity/.

[10] Newton, I. 1687. Philosophiae Naturalis Principia Mathematica. ("Mathematical Principles of Natural Philosophy”), London.

[11] Smith, G. 2007. "Isaac Newton.” Stanford Encyklopedia of Philosophy, Accessed Sep. 3, 2015. http://plato.stanford.edu/entries/newton/.

[12] Kala, T. "Comments on Matter/Mass/Field Categories”, Unitary Theory of the World, Accessed Sep. 3, 2015. http://www.tomas-kala.net/. 